\title{
Pixel Multichip Module Design for a High Energy Physics Experiment
}

\author{
G. Cardoso, J. Andresen, J.A. Appel, G. Chiodini, D. C. Christian, \\ B.K. Hall, S.W. Kwan, M.A. Turqueti, S. Zimmermann
}

\begin{abstract}
At Fermilab, a pixel detector multichip module is being developed for the $\mathrm{BTeV}$ experiment. The module is composed of three layers. The lowest layer is formed by the readout integrated circuits (ICs). The back of the ICs is in thermal contact with the supporting structure, while the top is flip-chip bump-bonded to the pixel sensor. A low mass flex-circuit interconnect is glued on the top of this assembly, and the readout IC pads are wire-bounded to the circuit. This paper presents recent results on the development of a multichip module prototype and summarizes its performance characteristics.
\end{abstract}

\section{INTRODUCTION}

At Fermilab, the BTeV experiment has been approved for the $\mathrm{C}$-Zero interaction region of the Tevatron [1]. One of the tracker detectors for this experiment will be a pixel detector composed of 62 pixel planes of approximately 100x100 mm each, assembled perpendicular to the colliding beam and installed a few millimeters from the beam.

The planes in the pixel detector are formed by sets of different lengths of pixel-hybrid modules, each composed of a single active-area sensor tile and of one row of readout ICs. The modules on opposite faces of the same pixel station are assembled perpendicularly in relation to each other, as shown in Fig. 1.

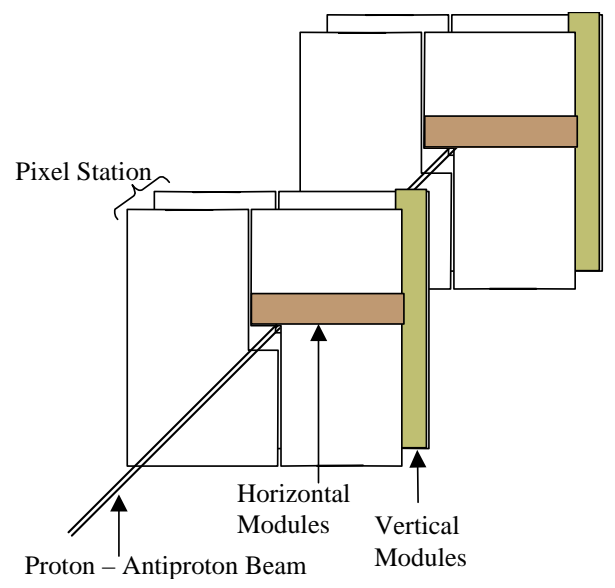

Fig. 1. Pixel detector stations

Operated by Universities Research Association Inc. under Contract No. DE-AC02-76CH03000 with the United States Department of Energy. FERMILAB-Conf-03/360-E.

G. Cardoso, J. Andresen, J.A. Appel, D.C. Christian, B.K. Hall, S.W. Kwan, M. Turqueti are with Fermi National Accelerator Laboratory, Batavia, IL 60510 USA.

S. Zimmermann is with Lawrence Berkeley Laboratory, Berkeley, CA 94720 USA.
The BTeV pixel detector module is based on a design relying on a hybrid approach [2]. With this approach, the readout chip and the sensor array are developed separately and the detector is constructed by flip-chip mating the two together. This approach offers maximum flexibility in the development process, the choice of fabrication technologies, and the choice of sensor material.

The multichip modules must conform to special requirements dictated by $\mathrm{BTeV}$ : the pixel detector will be inside a strong magnetic field (1.6 Tesla in the central field), the flex circuit and the adhesives cannot be ferromagnetic, the pixel detector will also be placed inside a high vacuum environment, so the multichip module components cannot outgas, the radiation rates (around $3 \mathrm{Mrad}$ per year) and temperature $\left(-5^{\circ} \mathrm{C}\right)$ also impose severe constraints on the pixel multichip module packaging design.

The pixel detector will be employed for on-line track finding for the lowest level trigger system and, therefore, the pixel readout ICs will have to read out all detected hits. This requirement imposes a severe constraint on the design of the readout $\mathrm{IC}$, the hybridized module, and the data transmission to the data acquisition system.

Several factors impact the amount of data that each IC needs to transfer: readout array size, distance from the beam, number of bits of pulse-height analog to digital converter (ADC) data format, etc. Presently, the most likely dimension of the pixel chip array will be 128 rows by 22 columns and 3 bits of ADC information.

\section{PiXel Module Readout}

The pixel module readout must allow the pixel detector to be used in the lowest level experiment trigger. Our present assumptions are based on simulations that describe the data pattern inside the pixel detector [3]. The parameters used for the simulations are: luminosity of $2 \times 10^{32} \mathrm{~cm}^{-2} \mathrm{~s}^{-1}$ (corresponds to an average of 2 interactions per bunch crossing), pixel size of $400 \times 50 \mathrm{~mm}^{2}$, threshold of $2000 \mathrm{e}^{-}$and a magnetic field of 1.6 Tesla.

Fig. 2 shows a sketch of the 40 chips that may compose a pixel half plane and the data rate for the station in the middle of 31 stations. The beam passes at the place represented by the black dot. 


\begin{tabular}{|c|c|c|c|c|c|c|c|c|c|}
\hline Module $1 \rightarrow$ & 11 & 13 & 17 & 17 & 20 & 18 & 16 & 13 & 8 \\
\hline Module $2 \rightarrow$ & 11 & 18 & 26 & 31 & 39 & 33 & 25 & 18 & 12 \\
\hline Module $3 \rightarrow$ & 16 & 20 & 37 & 61 & 76 & 59 & 39 & 26 & 18 \\
\hline Module $4 \rightarrow$ & 17 & 35 & 63 & 141 & 234 & 130 & 65 & 36 & 16 \\
\hline Module $5 \rightarrow$ & 23 & 35 & 74 & 234 & & & & & \\
\hline
\end{tabular}

Fig. 2. Average bit data rate at middle station, in Mbit/s

These numbers assume the 23-bit data format shown in Fig. 3. Table 1 presents the required bandwidth per module. From this table we see that each half-pixel plane requires a bandwidth of approximately $1.8 \mathrm{Gbit} / \mathrm{s}$.

\begin{tabular}{|l|l|l|l|}
22 & \multicolumn{2}{c|}{0} \\
\hline ADC & Beam Crossing Number & Column & Row \\
\hline
\end{tabular}

Fig. 3. Pixel module data format (23 bits)

Table 1. Half plane required bandwidth, in Mbit/s

\begin{tabular}{|c|c|}
\cline { 2 - 2 } \multicolumn{1}{c|}{} & Req. Bandwidth \\
\hline Module 1 & 133 \\
\hline Module 2 & 213 \\
\hline Module 3 & 352 \\
\hline Module 4 & 737 \\
\hline Module 5 & 366 \\
\hline Total & $\mathbf{1 8 0 1}$ \\
\hline
\end{tabular}

We've used simulations of the readout architecture with a clock of $35 \mathrm{MHz}$. This frequency can support a readout efficiency of approximately $98 \%$ when considering three times the nominal hit rate for the readout ICs closest to the beam. Efficiency is lost either due to a pixel being hit more than once before the first hit can be read out, or due to bottlenecks in the core circuitry.

\section{A. Proposed Readout Architecture}

The readout architecture is a direct consequence of the $\mathrm{BTeV}$ detector layout. The $\mathrm{BTeV}$ detector covers the forward direction, 10-300 mrad, with respect to both colliding beams. Hence, the volume outside this angular range is outside the active area and can be used to house heavy readout and control cables without interfering with the experiment. The architecture takes advantage of this consideration.

The data combiner board (DCB) located approximately 10 meters away from the detector remotely controls the pixel modules. All the controls, clocks and data are transmitted between the pixel module and the DCB by differential signals employing the low-voltage differential signaling (LVDS) standard. Common clocks and control signals are sent to each module and then bussed to each readout IC. All data signals are point to point connected to the DCB. Fig. 4 shows a sketch of the proposed readout architecture. For more details refer to [4].

This readout technique requires the design of just one radhard chip: the pixel readout IC. The point-to-point data links minimize the risk of an entire module failure due to a single chip failure and eliminate the need for a chip ID to be embedded in the data stream. Simulations have shown that this readout scheme results in readout efficiencies that are sufficient for the $\mathrm{BTeV}$ experiment.

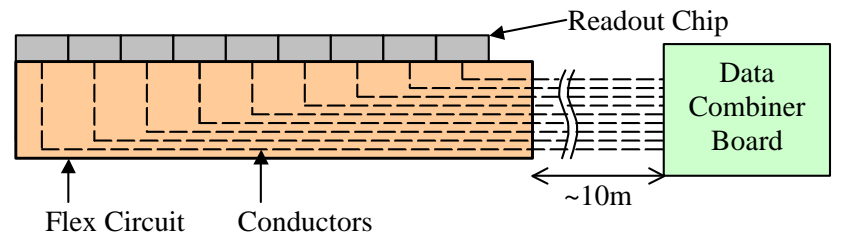

Fig. 4. Pixel module point-to-point connection

\section{PIXEL Module Prototype}

Fig. 5 shows a sketch of the pixel module prototype. This design uses the FPIX1 version of the Fermilab pixel readout IC.

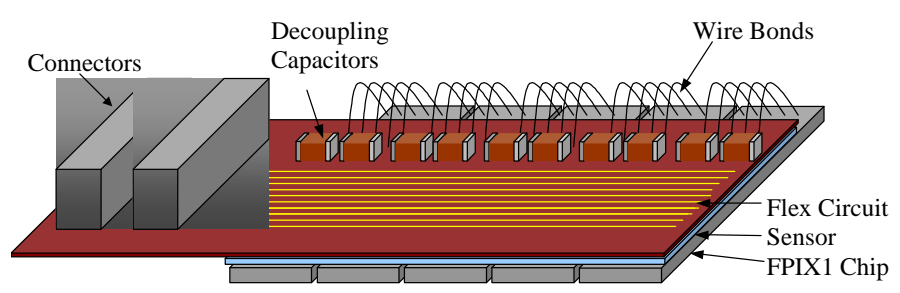

Fig. 5. Sketch of the pixel multichip module

The pixel multichip module prototype is composed of three layers, as depicted in Fig. 6. The pixel readout chips form the bottom layer. The back of the chips is in thermal contact with the station supporting structure, while the other side is flip-chip bump bonded to the silicon pixel sensor. The clock, control, and power pad interfaces of FPIX1 extend beyond the edge of the sensor.

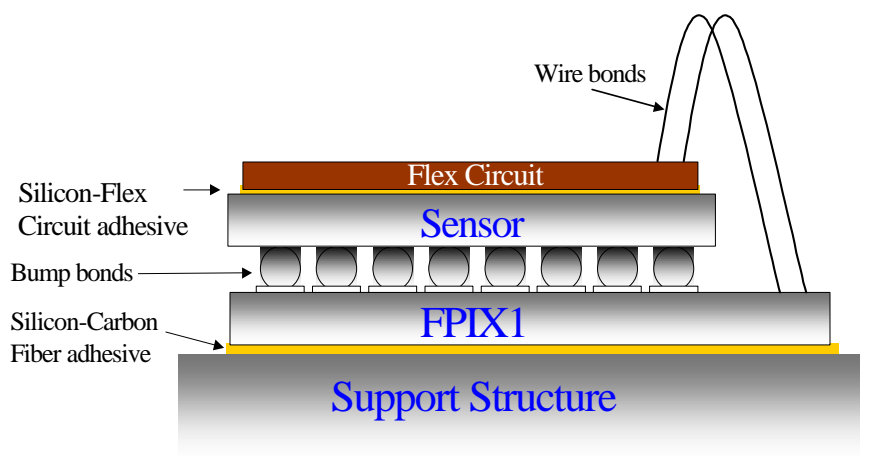

Fig. 6. Sketch of the pixel multichip module "stack"

The interconnect circuitry (flex circuit) is placed on the top of this assembly and the FPIX1 pad interface is wire-bonded to the flex circuit. The circuit then extends to one end of the 
module where low profile connectors interface the module to the data acquisition system. The large number of signals in this design imposes space constraints and requires aggressive design rules, such as $35 \mu \mathrm{m}$ trace width and trace-to-trace clearance of $35 \mu \mathrm{m}$.

This packaging requires a flex circuit with four layers of copper traces (as sketched in Fig. 7). The data, control and clock signals use the two top layers, power uses the third layer and ground and sensor high voltage bias use the bottom layer. The flex circuit has two power traces, one analog and one digital. These traces are wide enough to guarantee that the voltage drop from chip to chip is within the FPIX1 $\pm 5 \%$ tolerance. The decoupling capacitors in the flex circuit are close to the pixel chips. The trace lengths and vias that connect the capacitors to the chips are minimized to reduce the interconnection inductance. A picture of the flex circuit made by CERN is shown in Fig. 8.

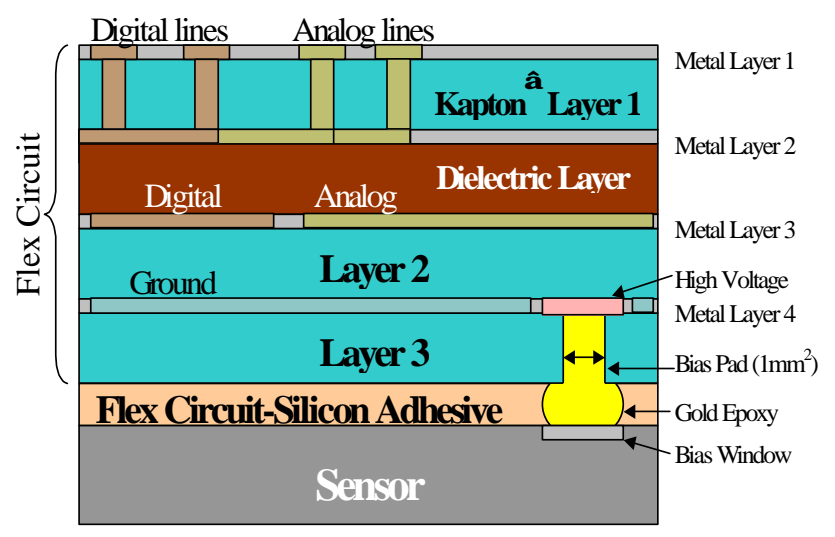

Fig. 7. Sketch of flex circuit cross section

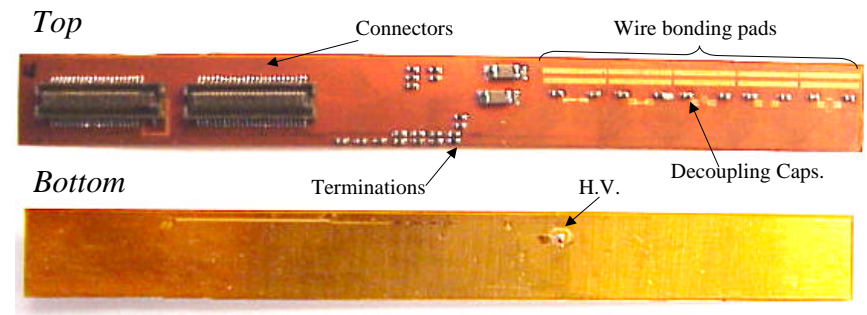

Fig. 8. Flex circuit picture

To minimize coupling between digital and analog elements, signals are grouped together into two different sets. The digital and analog traces are laid out on top of the digital and analog power supply traces, respectively. Furthermore, a ground trace runs between the analog set and the digital set of traces.

\section{A. High Voltage Bias}

The pixel sensor is biased with up to 1000 VDC through the flex circuit. The coupling between the digital traces and the bias trace has to be minimized to improve the sensor noise performance. To achieve this, the high voltage trace runs in the fourth metal layer (ground plane, see Fig. 7), and bellow the analog power supply trace. The high voltage is electrically connected to the sensor bias window with gold epoxy. An insulator layer in the bottom of the flex circuit isolates the ground in the fourth metal layer of the flex circuit from the high voltage of the pixel sensor.

\section{B. Assembly}

The interface adhesive between the flex circuit and the pixel sensor has to compensate for mechanical stress due to the coefficient of thermal expansion mismatches between the flex circuit and the silicon pixel sensor. Two alternatives are being pursued. One is 3M's thermally conductive tape [5]. The other is the silicone-based adhesive used in [6].

The present pixel module prototypes were assembled using $3 \mathrm{M}$ 's tape with a thickness of $0.05 \mathrm{~mm}$. Before mounting the flex circuit onto the sensor, a set of dummies with bump-bond structures where used to evaluate the assembly process. This assembly process led to no noticeable change in the resistance of the bumps. Fig. 9 shows a picture of the dummy.

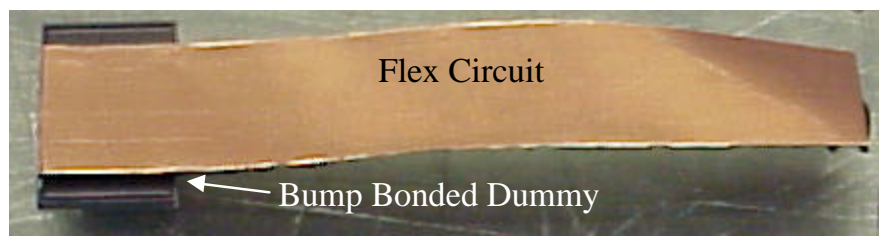

Fig. 9. Dummy bump bond structure

\section{PIXEL MODULE EXPERIMENTAL RESUlTS}

Two single pixel modules and one 5-chip module were characterized. One of these modules is a single readout IC (FPIX1) bump bonded to a SINTEF sensor (Fig. 10) using Indium bumps. In the second single pixel module the readout IC is not bump bonded to a sensor (Fig. 11). In all these prototypes the flex interconnect is located on the top of the sensor (as in the baseline design).

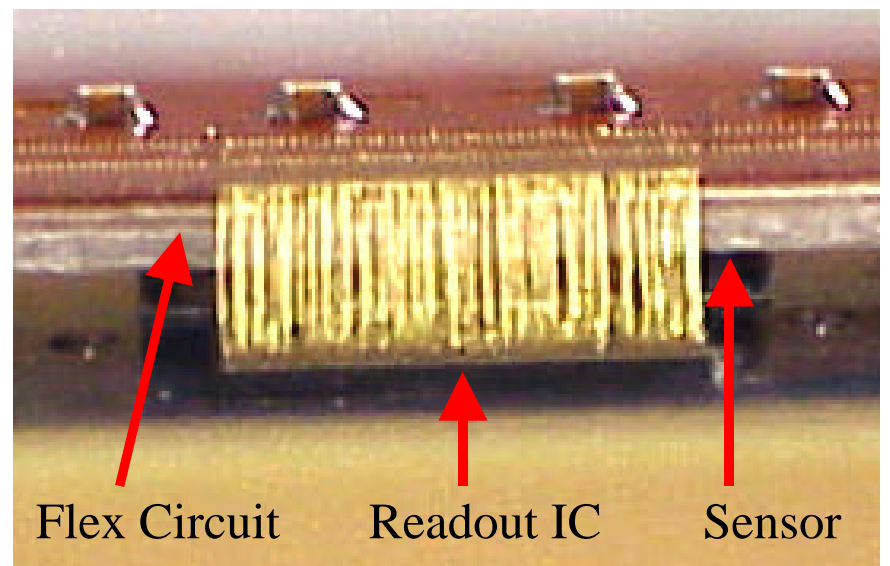

Fig. 10. Single chip bump-bonded to sensor 


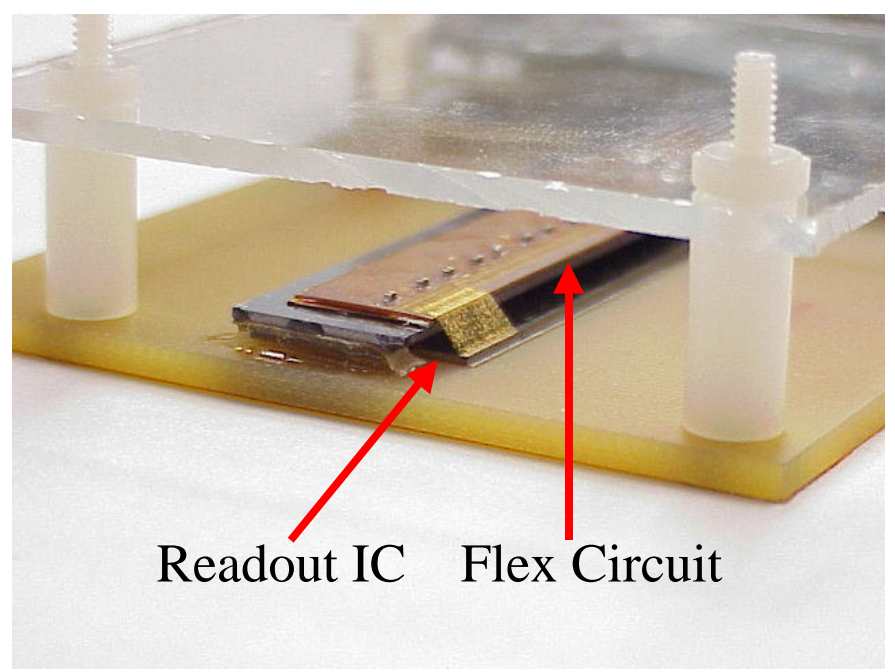

Fig. 11. Single chip without sensor

The pixel modules have been characterized for noise and threshold dispersion. These characteristics were measured by injecting charge in the analog front end of the readout chip with a pulse generator and reading out the hit data through a PCI based test stand. The results for different thresholds are summarized in Table 2.

Table 2. Performance of the pixel prototype modules (in $\mathrm{e}^{-}$)

\begin{tabular}{|c|c|c|c||c|c|c|c||}
\hline \multicolumn{4}{|c||}{ Without Sensor } & \multicolumn{4}{c||}{ With Sensor } \\
\hline \multicolumn{2}{|c|}{ Threshold } & \multicolumn{2}{|c|}{ Noise } & Threshold & \multicolumn{2}{c|}{ Noise } \\
\hline$\mu_{\mathrm{Th}}$ & $\sigma_{\mathrm{Th}}$ & $\mu_{\text {Noise }}$ & $\sigma_{\text {Noise }}$ & $\mu_{\mathrm{Th}}$ & $\sigma_{\mathrm{Th}}$ & $\mu_{\text {Noise }}$ & $\sigma_{\text {Noise }}$ \\
\hline 7365 & 356 & 75 & 7 & 7820 & 408 & 94 & 7.5 \\
\hline 6394 & 332 & 78 & 12 & 6529 & 386 & 111 & 11 \\
\hline 5455 & 388 & 79 & 11 & 5500 & 377 & 113 & 13 \\
\hline 4448 & 378 & 78 & 11 & 4410 & 380 & 107 & 15 \\
\hline 3513 & 384 & 79 & 12 & 3338 & 390 & 116 & 20 \\
\hline 2556 & 375 & 77 & 13 & 2289 & 391 & 117 & 21 \\
\hline \hline
\end{tabular}

The comparison of these results with previous results (single readout IC without the flex circuit on top) shows no noticeable degradation in the electrical performance of the pixel module. Fig. 12 shows the hit map of the pixel module with sensor using a radioactive source ( $\mathrm{Sr} 90)$, confirming that the bump bonds remain functional.

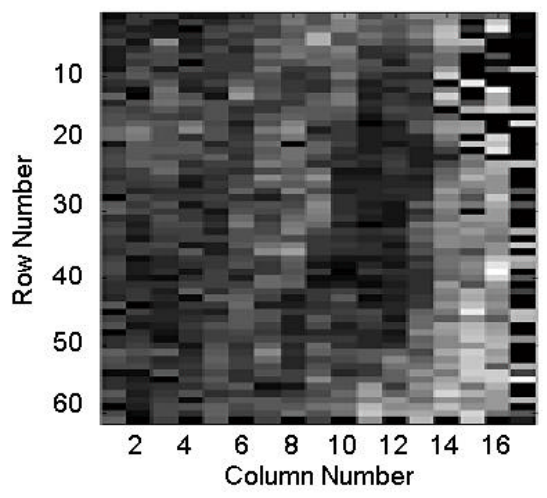

Fig. 12. Single pixel module hit map
The same set of tests was followed to characterize the 5-chip pixel module. Fig. 13 shows a picture of the pixel multichip module without the flex circuit, while Fig. 14 shows the complete module with flex circuit glued on top of the sensor. Table 3 summarizes the charge injection calibration results for all five chips for a given threshold. The hit map that of the 5chip module is shown in Fig. 15. This figure shows that chip 3 has a bad column, traced to be a digital problem with the readout IC.

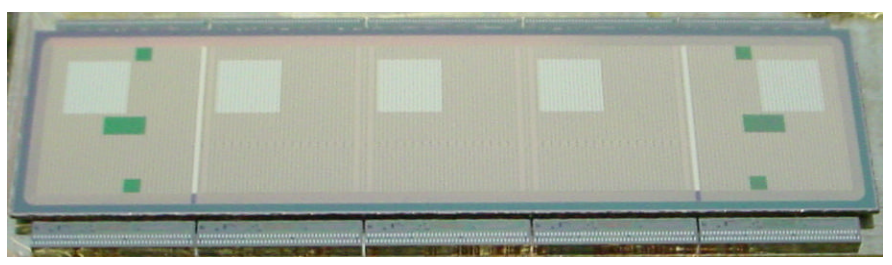

Fig. 13. Pixel module bump-bonded to 5 readout ICs

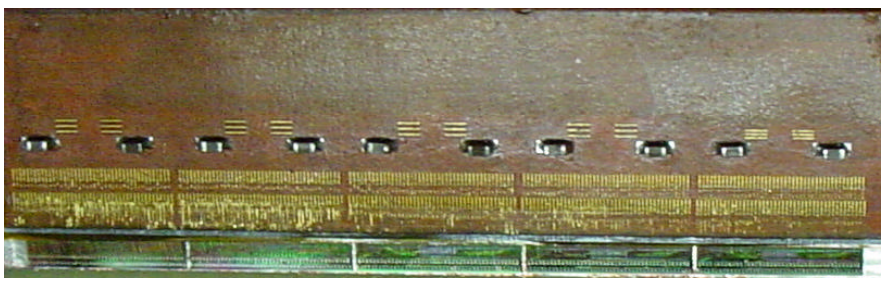

Fig. 14. 5-chip pixel module with flex circuit

Table 3. Performance of the pixel prototype module (in $\mathrm{e}^{-}$)

\begin{tabular}{||r|r|r|r|c||}
\hline \multirow{2}{*}{} & \multicolumn{2}{|c|}{ Threshold } & \multicolumn{2}{c|}{ Noise } \\
\cline { 2 - 5 } & $\mu_{\mathrm{Th}}$ & $\sigma_{\mathrm{Th}}$ & $\mu_{\text {Noise }}$ & $\sigma_{\text {Noise }}$ \\
\hline Chip 1 & 5360 & 360 & 260 & 19 \\
\hline Chip 2 & 5900 & 410 & 220 & 19 \\
\hline Chip 3 & 5250 & 400 & 230 & 19 \\
\hline Chip 4 & 5130 & 380 & 180 & 11 \\
\hline Chip 5 & 5020 & 420 & 240 & 20 \\
\hline
\end{tabular}

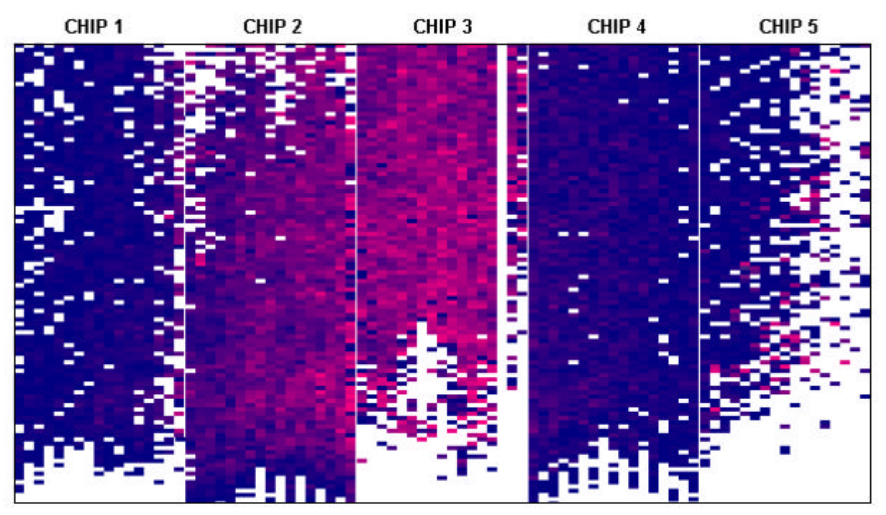

Fig. 15. Pixel multichip module hit map 


\section{RESUlTS OF THE HYBRIDIZATION TO PIXEL SENSORS}

The hybridization approach pursued offers maximum flexibility. However, it requires the availability of highly reliable, reasonably low cost fine-pitch flip-chip mating technology. We have tested three bump bonding technologies: indium, fluxed solder, and fluxless solder. Real sensors and readout chips were indium bumped at both the single chip and at the wafer level by BOEING, NA. Inc (Anaheim, CA) and Advance Interconnect Technology Ltd. (Hong Kong) with satisfactory yield and performance.

The bump bonding technologies with indium and solder are both viable for pixel detectors. The indium bumps look somewhat more susceptible to temperature variations. We visually observed the degeneration of these bumps at cold temperatures. The solder bumps on the other hand, were not affected by temperature changes or by radiation. For more details refer to [7].

\section{CONCLUSIONS}

We have described the baseline pixel multichip module designed to handle the data rate required for the $\mathrm{BTeV}$ experiment at Fermilab. The assembly process of a single chip and a 5-chip pixel module prototype was successful. The tests detected no crosstalk problems between the digital and analog sections of the readout chip and the flex circuit.
The characterization of the prototypes showed that there is no degradation in the electrical performance of the pixel module when compared with previous prototypes. Furthermore, the 5chip module showed no significant increase in noise and threshold dispersion when compared with the single chip prototypes.

\section{REFERENCES}

[1] Kulyavtsev, A., et al., BTeV Proposal, Fermilab, May 2000.

[2] Cardoso, G., et al., "Development of a high density pixel multichip module at Fermilab", $51^{\text {st }}$ ECTC, Orlando, Florida, May 28-31, 2001.

[3] Christian, D.C., et al., "Development of a pixel readout chip for BTeV," Nucl. Instr. and Meth. A 435, pp.144-152, 1999.

[4] Hall, B., et al., "Development of a Readout Technique for the High Data Rate BTeV Pixel Detector at Fermilab", Proceedings of the 2001 IEEE Nuclear Science Symposium, San Diego, November 4-10, 2001.

[5] Thermally Conductive Adhesive Transfer Tapes, Technical datasheet, 3M. April 1999

[6] Abt, I., et al., "Gluing Silicon with Silicone", Nucl. Instr. and Meth. A 411, pp. 191-196, 1998.

[7] Kwan, S., et al., "Study of Indium and Solder Bumps for the BTeV Pixel Detector", Proceedings of the 2003 IEEE Nuclear Science Symposium, Portland, October 19-24, 2003. 